\title{
An overview on preseismic anomalies in $L F$ radio signals revealed in Italy by wavelet analysis
}

\author{
Pier Francesco Biagi $\left({ }^{1}\right)\left({ }^{2}\right)$, Laura Castellana $\left({ }^{1}\right)$, Tommaso Maggipinto $\left({ }^{1}\right)$ and Anita Ermini $\left(^{3}\right)$ \\ ( $\left.{ }^{1}\right)$ Dipartimento di Fisica, Università degli Studi di Bari, Italy \\ $\left(^{2}\right)$ Centro Inter-Dipartimentale per la Valutazione e la Mitigazione del Rischio Sismico, \\ Università degli Studi di Bari, Italy \\ $\left.{ }^{3}\right)$ Dipartimento di Ingegneria dell'Impresa, Università degli Studi di Roma Tor Vergata, Roma, Italy
}

\begin{abstract}
Since 1996, the electric field strength of the two broadcasting stations MCO ( $f=216 \mathrm{kHz}$, southeast France) and CZE ( $f=270 \mathrm{kHz}$, Czech Republic) has been sampled every ten minutes by a receiver (AS) located in central Italy. Here, we review the results obtained by a detailed analysis applied to the data recorded from February 1996 up to December 2004. At first, the daytime and nighttime data were extracted and then, in the daytime data, the data collected in winter were separated from those collected in summer. On the second step the wavelet transform was applied. The results of this analysis are radio anomalies detected as earthquake precursors both for MCO and CZE data. In particular, regarding the MCO data, the main result was the appearance of a very clear anomaly during May-August 1998, at daytime and at nighttime. Such an anomaly can be considered as a precursor of a seismic sequence started on August 15, 1998 with 17 earthquakes $(M=2.2-4.6)$ on the Reatini mountains, a seismogenic zone located $30 \mathrm{~km}$ far from the AS receiver along the path MCO-AS. As concerns with the CZE data, the first result was obtained from the summer daytime data and it was the appearance of a very clear anomaly during August-September 1997, that can be considered a precursor of the two earthquakes with magnitude $M=5.6$ and $M=5.9$ that occurred on September 26 in the Umbria-Marche region (Central Italy). The second result was the appearance of an anomaly during February-March 1998, at daytime and at nighttime, that can be related to the preparatory phase of the strong $(M=5.1-6.0)$ Slovenia seismic sequence that occurred in a zone lying in the middle of the CZE-AS path.
\end{abstract}

Key words LF radio-waves - wavelet - seismicity - precursors

\section{Introduction}

Research into the interaction between seismic activity and disturbances in radiobroadcasts has been carried out for many years.

Mailing address: Dr. Pier Francesco Biagi, Dipartimento di Fisica, Università degli Studi di Bari, Via Amendola 173, 70125 Bari, Italy; e-mail: biagi@ fisica.uniba.it
Mainly, pre-seismic disturbances in radio waves that lie in the VLF (3-30 kHz) frequency band have been presented (Hayakawa and Sato, 1994; Hayakawa et al., 1996, 2002; Molchanov and Hayakawa, 1998). Recently, Rozhnoi et al. $(2004,2005,2006)$ revealed a convincing connection between the nightime anomalies in a LF $(40 \mathrm{kHz})$ radio signal and the earthquakes that occurred with $M \geq 5.0$ nearby the radio paths. Moreover, Molchanov et al. (2006) have presented possible seismic disturbances in VLF radio signals radiated by powerful transmitters located on the ground but recorded on board of the DEMETER satellite. The VLF-LF (10-60 $\mathrm{kHz}$ ) radio signals are used for time standard 
signal and worldwide navigation support and propagate in the earth-ionosphere wave-guide mode along great circle propagation paths. The analysis is based on the amplitude and the phase variations of the radio signals propagating from different transmitting stations. The anomalous variations in the radio signals are produced by perturbations in the ionosphere. These are related to the particles/gas/water release from the ground surface during the preparation phase of large earthquakes. The subsequent changes in the atmosphere are usually supposed to take place in a thin near ground layer with a thickness of several hundred meters. According to the most recent and convinc-

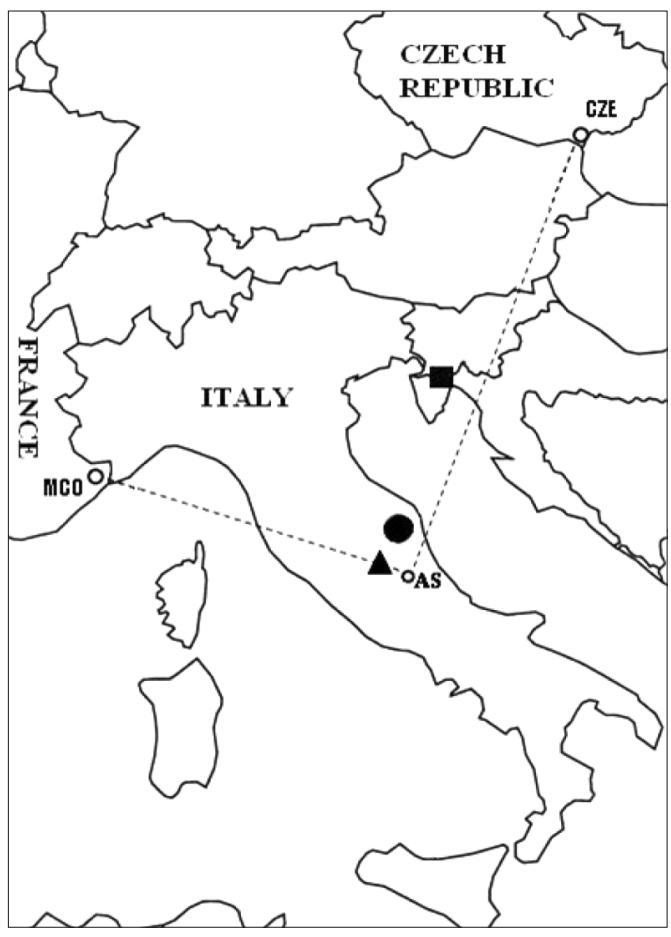

Fig. 2. Map showing the location of the receiver $\mathrm{AS}$ and of the two transmitters MCO and CZE. The two radio-paths are indicated by dotted lines. The location of the Reatini mountains seismogenetic area is shown by a black triangle. The black square represents the location of the Slovenia seismic sequence, and the black circle indicates the location of the $M=5.6$ and $M=5.9$ Umbria-Marche earthquakes. ing model (Mareev et al., 2002; Molchanov, 2004) the extension of the effect up to the ionosphere is due to atmospheric gravity waves of low amplitude generated during the quoted ground processes. These waves propagate up to the ionosphere, penetrate inside and interact with the ionosphere turbulence producing disturbances in the propagation of the VLF-LF radio waves.

In 1995 some of the authors designed and built a receiver able to measure the electric field strength of LF broadcasting stations. The receiver was put into operation at the entrance of a natural cave located in Central Italy (AS) and, on the basis of the best reception, the transmitting stations MCO (France, $f=216 \mathrm{kHz}$ ) and CZE (Czech Republic, $f=270 \mathrm{kHz}$ ) were selected. The respective transmitter-receiver distances are $518 \mathrm{~km}$ and $818 \mathrm{~km}$. In 1996 the measurements started, with a sampling frequency of ten minutes. Figure 1 shows the location of the transmitters and the receiver.

Looking for anomalous patterns in the raw or filtered data of both the radio signals, several anomalies were obtained (Bella et al, 1998; Biagi et al., 2001a,b; Biagi and Hayakawa, 2002; Biagi et al., 2003). Their features are decreases or increases in the electric field amplitude and they could be related to the seismic activity. Then, since the LF radio signals are strongly changeable during the day and during the cycle of the seasons, a different approach of analysis (Biagi et al., 2005; Biagi et al., 2006a,b) was carried out: after separating each data set of MCO and CZE radio signals in nighttime data and daytime data (in winter and summer), they were processed by means of the wavelet analysis.

Here we present an overview of the results obtained using the quoted technique of analysis to investigate the background of the signals and to detect possible anomalies. The MCO and CZE data sets collected from February 1996 to December 2004 were used.

\section{Theoretical computation and data analysis.}

It is well known that the $L F$ radio signals are characterised by the ground wave and the sky 


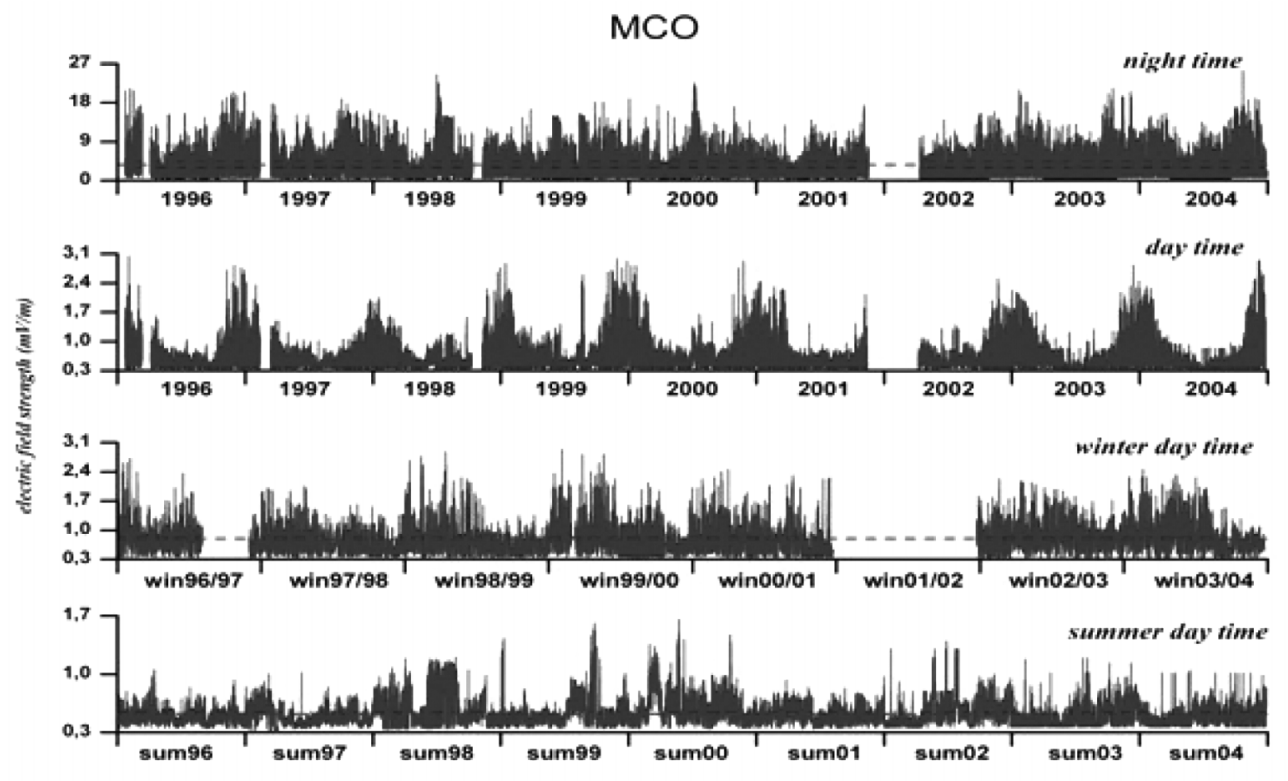

\section{CZE}
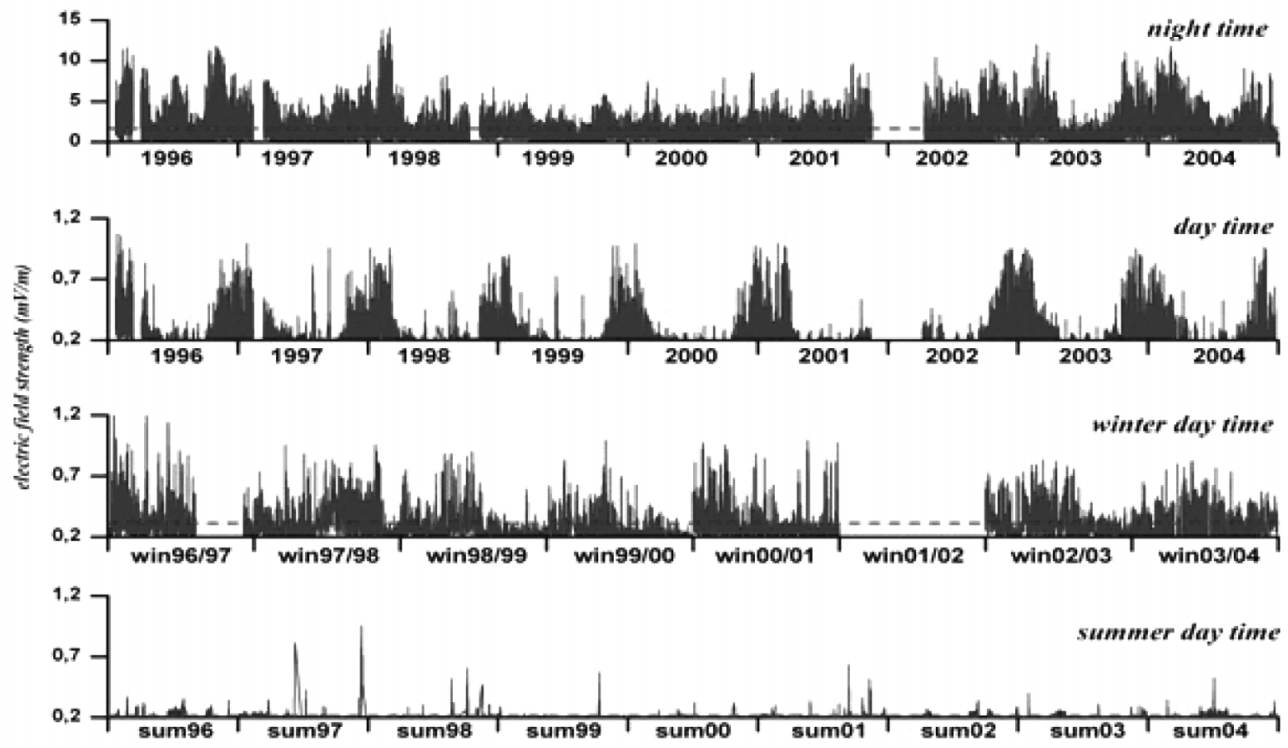

Fig. 2a,b. a) From the top reading downwards, the MCO electric field strength at night time (9.00-12.00 p.m., local solar time), at daytime (9.00 a.m.-2.00 p.m., local solar time), at winter daytime and at summer daytime, from February 1996 to December 2004. The dashed horizontal lines represent the mean values (table I) of the relative data set. The gaps in the trends correspond to interruptions in the data collection. b) The same of Figure $2 \mathrm{a}$ but with reference to the CZE electric field strength. 
Table I. Experimental (Exp) and theoretical (Theo) values of the electric field strength ( $\mathrm{mV} / \mathrm{m}$ ) for the MCO $(216 \mathrm{kHz})$ and $\mathrm{CZE}(270 \mathrm{kHz})$ radio signals at AS receiver.

\begin{tabular}{|c|c|c|c|c|}
\hline \multicolumn{5}{|c|}{ Electric field strength (experimental values) } \\
\hline Transmitter & Day winter & Day summer & Night & \\
\hline $\mathrm{MCO}$ & 0.79 & 0.52 & 3.65 & \\
\hline $\mathrm{CZE}$ & 0.31 & 0.21 & 1.65 & \\
\hline \multicolumn{5}{|c|}{ Sky wave } \\
\hline \multirow[t]{2}{*}{$\mathrm{MCO}$} & Theoretical values & 0.50 & 0.02 & 3.10 \\
\hline & Experimental values & 0.25 & - & 3.11 \\
\hline \multirow[t]{2}{*}{$\mathrm{CZE}$} & Theoretical values & 0.40 & 0.016 & 1.62 \\
\hline & Experimental values & 0.23 & - & 1.57 \\
\hline \multicolumn{5}{|c|}{ Ground wave (theoretical values) } \\
\hline $\mathrm{MCO}$ & 0.54 & & & \\
\hline $\mathrm{CZE}$ & 0.08 & & & \\
\hline
\end{tabular}

wave propagation modes. The ground wave provides a rather stable signal, while the sky wave varies widely from day to night and, at daytime, from winter to summer. The amplitude recorded by the receiver is the superimposition of the two waves but, for distances greater than $300-400 \mathrm{~km}$, the recorded electric field strength can be considered a simple addition of the two waves (CCIR, 1990a). So it was assumed that the $\mathrm{MCO}$ and the CZE amplitude is the addition of the ground wave and the sky wave contributions. To evaluate the two different contributions, the theoretical amplitudes of the ground wave and the sky wave have been calculated. Details of the theoretical computations are presented in Biagi et al. (2006a). As for the ground wave, the propagation of this wave over a smooth, homogeneous, curved Earth, with exponentially decreasing refractive index (Rotheram, 1981a,b) was considered; for the sky wave, using the wave hop model (Knight, 1973; CCIR, 1990b), the sky wave signal was supposed as a ray starting from the transmitter and reflected, with one or more hops, by the lower ionosphere and by the ground. Both the distances transmitter-receiver (MCO-AS and CZE-AS) are consistent with only one hop, whose reflection point on the ionosphere is in the middle of the radio path. Then, the following step of the analysis was carried out on the experimental data, by separating the daytime data from the nighttime ones for each radio signal. In order to obtain data sets related to daytime and to nighttime regardless of the season and with the same number of data per day, the range 09.00 a.m.-02.00 p.m. (local solar time) for the daytime and the range 09.00-12.00 p.m. (local solar time) for the nighttime was selected. This last choice is also due to an interruption of 3-4 $\mathrm{h}$ in the radio broadcasts for both MCO and CZE transmitters after midnight (local solar time $=\mathrm{UT}+1 \mathrm{~h}$ ). Then, only for the daytime data, the data collected in winter (December 21-March 21) were separated from the ones collected in summer (June 21-September 21). Figure 2 shows the nighttime, daytime, winter daytime and summer daytime trends of the $\mathrm{MCO}$ and the CZE electric field strength, from February 1996 to December 2004. The mean value for each data set is indicated by a horizontal dashed line. Finally, theoretical and experimental values resulting from this analysis are reported in table I. The mean experimental values of the sky wave have been obtained re- 
moving the theoretical value of the corresponding ground wave from the experimental values of the electric field strength.

Then, because of the changing-in-time statistical characteristics of the signals, the wavelet transform (Torrence and Compo, 1998) on the nighttime data and on the daytime data at winter and summer, was applied. In this way, it has been possible to highlight the spectral components of the signal by using variable-width time windows, by considering that the frequency content of these windows is inversely related to the time widths; so, the localization of the signal is simultaneously obtained both in time and in frequency (Daubechies, 1992; Strang and Nguye, 1996). In the analysis, we adopted as wavelet the «Morlet function» (Torrence and Compo, 1998). In this case the wavelet transform of a time signal is a complex series that can be usefully represented by its square amplitude, i.e. we consider the so called wavelet power spectrum. The power spectrum is a $2 \mathrm{D}$ plot that, once properly normalized with respect to the power of the white noise, gives informa- tion on the strength and precise time occurrence of the various Fourier components which are present in the original time series. Instead of reporting the entire 2D plot, we consider $i$ in figs. 3 and 4 the wavelet power corresponding to the Fourier period in which the maximum power is found, and hence the plot is 1D representation of a single period with respect to time. Figure 3 makes reference to $\mathrm{MCO}$ radio signal and shows a clear anomaly during May-August 1998, at daytime and at nighttime. Figure 4 makes reference to the $\mathrm{CZE}$ radio signal and two main results stand up: the first one is an evident anomaly during August-September 1997 at summer daytime, as shown in fig. $4 \mathrm{a}$; the second one is an anomaly appearing during February-March 1998 mainly at nighttime as shown in fig. $4 b$.

We have named as (a), (b), and (c) the previous three anomalies, where (a) is the one revealed in the MCO radio signal, (b) and (c) the anomalies pointed out in the CZE radio signal, occurred in summer 1997 and in winter 1998, respectively.

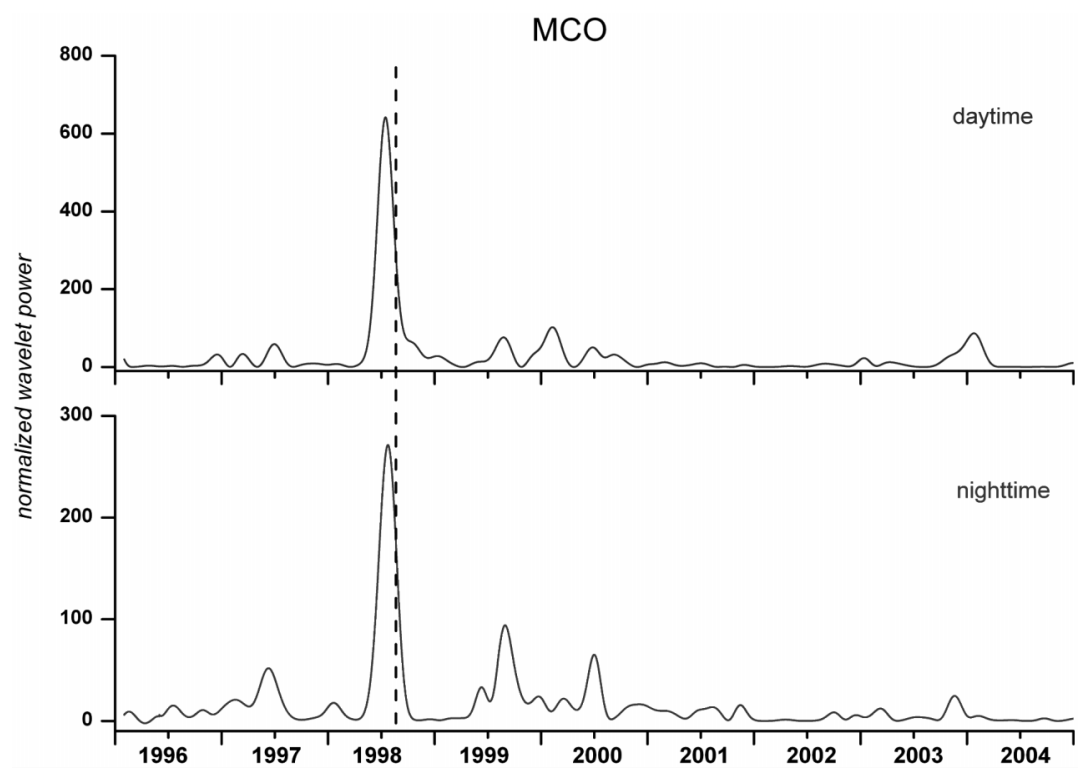

Fig. 3. Normalized wavelet power trend from February 1996 to December 2004 of the MCO electric field strength at daytime and at nighttime for the period 50 days that corresponds to the maximum power. The dashed vertical line indicates the time occurrence of the Reatini mountains main shock $(M=4.6)$. 


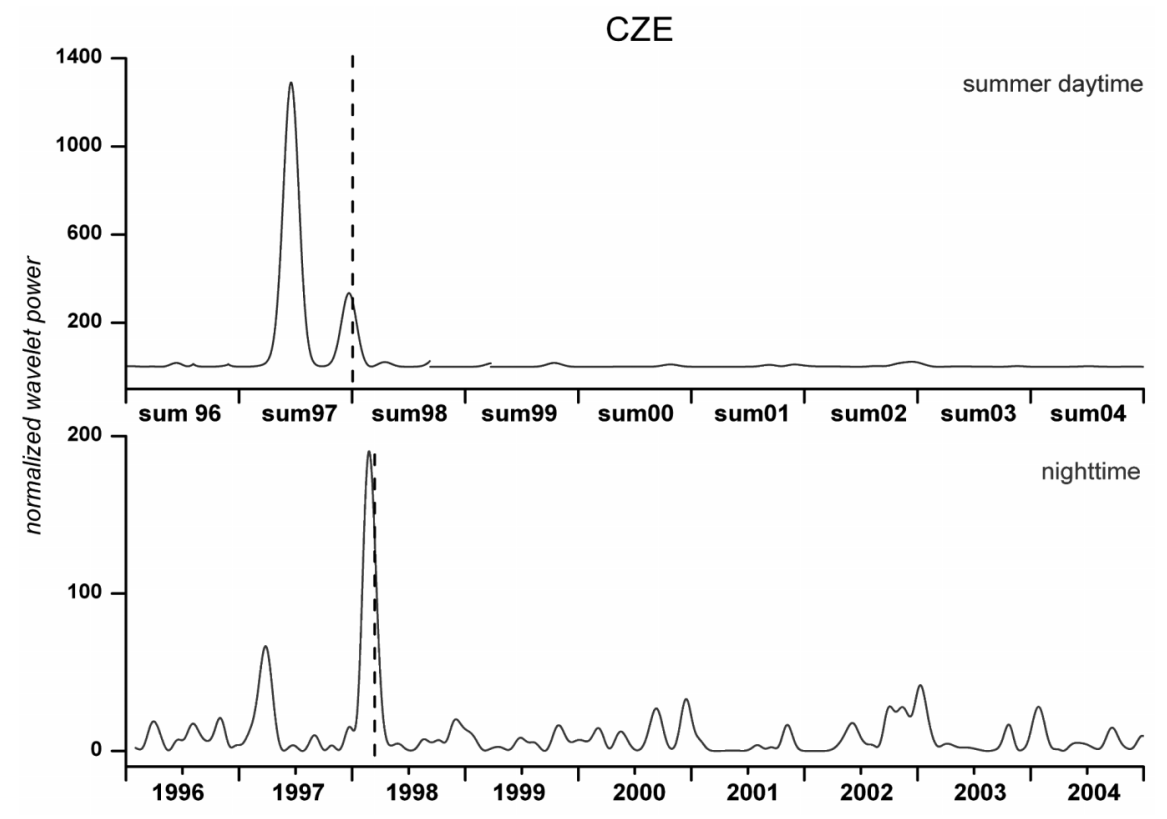

Fig. 4a,b. a) Normalized wavelet power spectrum from 1996 to 2004 of the CZE electric field strength in summer daytime (June 21-September 21 of every year) for the period 10 days that corresponds to the maximum power. The dashed vertical line represents the time occurrence of the two earthquakes $(M=5.6$ and $M=5.9)$ happened on September 26, 1997 in the Umbria-Marche region. b) Normalized wavelet power spectrum from February 1996 to September 2004 of the CZE electric field strength at nighttime for the period 20 days that corresponds to the maximum power. The dashed vertical line represents the time occurrence of the first Slovenia earthquake $(M=5.2$, March 13, 1998).

It must be noted that none of the previous anomalies ever appears in either the MCO or CZE radio signals.

\section{Discussion}

Taking into account the results reported in table I and considering that the receiver has an instrumental zero equal to $0.20 \mathrm{mV} / \mathrm{m}$, it is possible to make the following statements: 1 ) for both the $\mathrm{MCO}$ and CZE radio signals, the sky wave at nighttime and at winter daytime can be recorded; on the contrary, at summer daytime its value is below the instrumental zero; 2 ) the mean value of the sky wave at nighttime is the same in winter and summer; 3 ) for the MCO radio signal, the experimental value at summer daytime represents, practically, the intensity of the ground wave; for the CZE signal, the theoretical value of the ground wave is below the instrumental zero, so that the experimental value recorded at summer daytime does not represent any signal, but only some noise. Mainly its origin is anthropic because related to summer storms (lightnings). Such an activity generally produces $0.4-0.6$ $\mathrm{mV} / \mathrm{m}$ spikes with some hour duration at most and it can be checked by the data of a local meteorological station.

Now, the anomalies pointed out in the previous section will be examined.

\subsection{Anomalies (a) and (b)}

The radio anomaly (a) is represented (fig. 3) by an increase in the electric field strength, both at daytime and at nighttime, mainly in summer: 
so, from the previous considerations, the sky wave cannot be involved. On the contrary, it suggests the possibility that the anomaly is related to an increase in the ground wave propagation mode and it can be investigated on the daytime data. Looking at the summer daytime data set (fig. 2a), large signals over $0.8-1.0 \mathrm{mV} / \mathrm{m}$ appear during summer 1998: their comparison with the ground wave theoretical value at the AS receiver (table I) shows a $50 \%$ increase of the ground wave electric field intensity.

Anomaly (b) observed in the CZE radio signal in summer 1997 discloses two large signals with a duration of 10-20 days in the trend at the bottom of fig. $2 \mathrm{~b}$; these signals start on July 30 and on September 14, respectively. Their amplitudes reach peak values up to $0.8 \mathrm{mV} / \mathrm{m}$. In those periods, summer storms (lightnings) were not revealed by the data of a local meteorological station. The anomaly revealed by the wavelet analysis (fig. 4a) is produced mainly by the quoted large signals. Taking into account that the anomaly appears only at summer daytime, the sky wave cannot be involved and so, as for anomaly (a), a connection with an increase in the ground wave propagation mode can be proposed. Such an increase could produce a recordable signal at summer daytime, when, normally, the elecric field strength cannot be recorded, being below the instrumental zero (table I). Otherwise, no anomaly can be revealed at nighttime, when the ground wave is superimposed on the sky wave that is much more intense.

Now it is necessary to explain the processes that can produce the quoted increases. Several parameters control the ground wave propagation of a radio signal: the scale height of the troposphere, its refractive index, the relative permittivity and the conductivity of the ground. The ground conductivity and the refractive index of the troposphere are the most influential and the electric field strength of the ground wave increases if the value of one or both the previous parameters increases along the radio path or in some zone of it. At first, the meteorological conditions and the rainfall in the areas of the radio paths were checked and no anomalous situations emerged during the two time periods when the anomalies occurred.

\subsubsection{Connection of the anomalies (a) and (b) with the seismic activity}

From the Seismic Bulletins of the INGV (National Institute of Geophysics and Volcanology) it results that the anomaly (a) occurred before the start (August 15, 1998) of a seismic sequence with 17 earthquakes $(M=2.2-4.6)$, in a zone located $30 \mathrm{~km}$ far from the AS receiver, along the MCO-AS radio path. The sequence is located in the seismogenetic area of the Reatini Mountains, in the central Apennines (Figure 1), that is approximately a rectangle, with a diagonal $40 \mathrm{~km}$ long in the northeast direction. The quoted seismic sequence is the only significant event occurred in the first Fresnel zone of the MCO radio signal, that is considered (Hayakawa et al., 1996; Molchanov and Hayakawa, 1998; Rozhnoi et al., 2004) the zone sensitive to the seismo-atmospheric effects for earthquakes up to $M=5.0$. In the light of these considerations, the anomaly (a) was considered a precursor of the Reatini Mountains seismic sequence. Looking at fig. 3, the precursor started about 90 days before the occurrence of the sequence.

As regards anomaly (b), it occurred before the two $(M=5.6$ and $M=5.9)$ earthquakes happened on September 26, 1997 in the UmbriaMarche (Central Italy) region. Their location is indicated in fig. 1. These earthquakes started an intense seismic sequence lasting more than six months. From the Seismic Bulletins they result the only strong and significant events that can affect the CZE radio signal in 1997. So, we considered anomaly (b) as a precursor of the Umbria-Marche earthquakes. From fig. 4a a total premonitory time of about 60 days can be assumed. The following step of the study has been performed to justify the connection between the quoted two radio anomalies and the related seismic activity. As mentioned above, the most influential parameters on the ground wave propagation are the conductivity of the ground and the refractive index of the troposphere. The first is strongly affected by the water content and in many cases a small increase in the percentage of water is enough to step up the conductivity enormously (Telford et al., 2002). The refractive index of the troposphere 
varies with the height, mainly, and particularly we have to take into account the value of the refractive index at the surface of the ground (CCIR, 1990c): it is affected by the chemical composition of the air and it changes when variations, also the small ones, happen in this composition. So, in the light of these considerations, we propose that during the preparatory phase of the earthquakes, an increase in the underground waters in the surface layers and emissions of gases, particles and so on (atmosphere-litosphere coupling) happened in a wide zone, producing a consequent increase in the local conductivity of the ground and of the refractive index at the surface, respectively. Such modifications produced anomalous zones along the MCO-AS and CZE-AS paths able to increase the electric field strength of the ground wave at the AS receiver. In order to reproduce the increase in the intensity of the ground wave, as pointed out in anomalies (a) and (b), the theoretical intensity was evaluated using values for the ground conductivity and the surface refractive index, realistic but larger than the normal ones (CCIR, 1990c and 1990d). These values were introduced in zones defined as it follows: for the MCO radio signal, in a path $40 \mathrm{~km}$ long in correspondence with the Reatini mountains area (fig. 1); for the CZE radio signal, in the 75 $\mathrm{km}$ long radio path in Italy (fig. 1).

In such a way, for the MCO intensity of the ground wave, a value of $0.80 \mathrm{mV} / \mathrm{m}$ was obtained, i.e. an increase of about $50 \%$ with respect to the standard value reported in table I; this value is consistent with the anomalous signals revealed on summer data in 1998, i.e. with the anomaly (a).

For the CZE intensity of the ground wave the value $0.34 \mathrm{mV} / \mathrm{m}$ was obtained. Such a value is a recordable value with respect to the negligible theoretical one (table I), but much less than the intensity of the signals responsible for the anomaly (b). So, in this case, a second model was proposed. The ground wave propagation of a radio signal can be affected by ducts, reflecting layers, scattering zones and so on in the troposphere (CCIR, 1990d; Crane, 1981; Rue, 1987) as a consequence of emissions of gases, particles and so on, during a preseismic phase. So, if a tropospheric reflecting layer or a tro- pospheric duct is supposed to lay in the zone of the Umbria-Marche earthquakes, then reflected rays can arrive in the different zones and they can interact with each other producing interference. So, the radio signal level is very high in the constructive interference areas, while in the destructive ones is very low. If the formation of an area of constructive interference nearby the AS receiver is assumed, the existence of the large CZE signals appeared in summer daytime data in 1997 can be justified. This last model rather than the previous one, could be taken into account to justify the anomaly (b). This anomaly seems to be formed by two successive phases (fig. 4a) and it means that the irregularities (ducts, reflecting layers, scattering zones) in the troposphere claimed by the model, occurred twice, each about 30 days long, rather than only once but with a longer duration (60 days). It must be noted that taking into account the atmospheric circulation and the currents, a short duration of the quoted irregularities in the troposphere is more realistic respect of a long duration.

\subsection{Anomaly (c)}

The last anomaly (c) under study appeared in the CZE radio signal, in winter 1998, mainly in nighttime data. So, on the basis of the first statements of the discussion, it is related to the sky wave, showing exaggerated intensity.

The anomaly is an increase in the intensity of the radio signal (fig. $4 \mathrm{~b}$ ) and at its end a strong seismic sequence $(M=5.1-6.0)$ started in Slovenia, about in the middle of the transmitterreceiver path (fig. 1). So, the radio anomaly can be considered a precursor of these earthquakes and a premonitory time of about 50 days (fig. 4b) can be assumed. It must be noted that this anomaly was also pointed out with a different methodology presented in Biagi and Hayakawa (2002).

According to the wave hop theory, the exaggerated intensity of the sky wave may be explained by a 2-3 fold increase in the reflection coefficient of the ionosphere, just in the zone lying above the middle point of the CZE-AS path. This increase should be a consequence of 
the emission of radon, ions, electromagnetic waves, etc. (Biagi and Hayakawa, 2002; Hayakawa et a., 1996; Morgounov et al., 1994) from the focal zone of the forthcoming earthquake. Also a different model, claiming an exaggeration of the atmospheric tides as consequence of gravity changes in the focal zone has been proposed (Biagi et al., 2003)

\section{Conclusions}

This study disclocled that the LF radio signals can supply two different types of information related to the ground wave and to the sky wave. In particular, anomalies appearing mainly in the ground wave could be related to variations in some parameters of the ground and/or of the troposphere, which control the ground wave propagation mode. On the other hand, anomalies mainly appearing in the sky wave seem to be connected with disturbances in the ionosphere. In both cases, such variations can be produced by the processes that take place during the preparatory phase of earthquakes, according to the atmosphere-litosphere coupling model.

\section{Acknowledgements}

The seismic data used in this paper were obtained by the INGV (Istituto Nazionale di Geofisica e Vulcanologia) web site (http://www.ingv.it).

The authors are very grateful to V. Lapenna and to an anonymous Referee for their helpful suggestions to enhance the paper.

\section{REFERENCES}

Bella, F., P.F. Biagi, M. Caputo, E. Cozzi, G. Della Monica, A. ERmini, W. Plastino and V. Sgrigna (1998): Field strength variations of LF radio waves prior to earthquakes in Central Italy, Phys. Earth Planet. Int., 105, 279-286.

Biagi, P.F. and M. HAYAKaWA (2002): Possible premonitory behaviour of LF radiowaves on the occasion of the Slovenia earthquakes $(M=5.2-6.0-5.1)$ occurred on March-May 1998, in Seismo Electromagnetics: Lithosphere-Atmosphere-Ionosphere Coupling (Eds. M. HAYAKAWA and O. MOLCHANOV), (TERRAPUB, Tokyo), 249-253.
Biagi, P.F., R. Piccolo, A. Ermini, S. Martellucci, C. Bellecci, M. Hayakawa, V. Capozzi and S.P. KingsLEY (2001a): Possible earthquake precursors revealed by LF radio signals, NHESS, $\mathbf{1}(1-2)$.

Biagi, P.F., R. Piccolo, A. Ermini, S. Martellucci, C. Bellecci, M. Hayakawa and S.P. Kingsley (2001b): Disturbances in LF radio-signals as seismic precursors, Ann. Geofis., 44 (5/6),

Biagi, P.F., R. Piccolo, V. CAPOZZI, A. ERmini, S. MartelLUCCI and C. BELLECCI (2003): Exalting in atmospheric tides as earthquake precursor, NHESS, 3, 197-201.

Biagi, P.F., L. Castellana, T. Maggipinto, R. Piccolo, A Minafra, A. Ermini, S. Martellucci, C. Bellecci, G. Perna, V. Capozzi, O.A. Molchanov and M. HAYAKAWA (2005): A possible preseismic anomaly in the ground wave of a radio broadcasting $(216 \mathrm{kHz})$ during July-August 1998 (Italy), NHESS, 5, 727-732.

Biagi, P.F., L. Castellana, T. Maggipinto, A. ERmini, G. PERNA and V. CAPOZZI (2006a): Electric field strength analysis of 216 and $270 \mathrm{kHz}$ broadcast signals recorded during 9 years, Radio Sci., 41.

Biagi, P.F., L. Castellana, T. Maggipinto, R. Piccolo, A. Minafra, A. Ermini, S. Martellucci, C. Bellecci, G. Perna, V. Capozzi, O.A. Molchanov and M. HAYAKAWA (2006b): LF radio anomalies revealed in Italy by the wavelet analysis: possibile preseismic effects during 1997-1998, Phys. Chem. Earth, 31, 403408.

CCIR-International Radio Consultative Committee (1990a): Method for predicting sky wave field strengths at frequencies between $150 \mathrm{kHz}$ and 1705 kHz, Rep. 575-4, 167-184.

CCIR-International Radio Consultative Committee (1990b): Sky-wave propagation and circuit performance at frequencies between about $30 \mathrm{kHz}$ and 500 kHz, Rep. 265-7, 212-229.

CCIR-International Radio Consultative Committee (1990c): World atlas of ground conductivities, Rep. 717-3, 95-105.

CCIR-International Radio Consultative Committee (1990d): Effects of tropospheric refraction on radiowave propagation, Rep. 717-3, 95-105.

CRANE, R.K. (1981): A review of transhorizon propagation phenomena, Radio Sci., 16 (5), 649-669.

DAUBECHIES, I. (1992): Ten lectures on wavelets, CBMS NSF Regional Conferences Series in Applied Mathematics (SIAM, Philadelphia, PA), 61

HAYAKAWA, M. and H. SATO (1994): Ionospheric perturbations associated with earthquakes as detected by subionospheric VLF propagation, in Electromagnetic Phenomena Related to Earthquake Prediction, edited by M. HAYAKAWA and Y. FuJinawa (Terrapub, Tokyo), 391-397.

HaYAKaWa, M., O.A. Molchanov, T. ONDOH and E. KaWAI (1996): The precursory signature effect of the Kobe earthquake on VLF subionospheric signals, J. Comm. Res. Lab., 169-180.

Hayakawa, M., O.A. Molchanov, N. Shima, A.V. ShVets and N. Yамамото (2002): Wavelet analysis of disturbances in subionospheric VLF propagation correlated with earthquakes, in Seismo Electromagnetics: Lithosphere-Atmosphere-Ionosphere Coupling, edited by M. HaYAKaWA and O. Molchanov (Terrapub, Tokyo), 223-228. 
KNIGHT, P. (1973): MF propagation: a wave-hop method for ionospheric field-strength prediction, $B B C$ Eng., 10, 22-34.

MareEv, E.A., D.L. Ludin and O.A. Molchanov (2002): Mosaic source of internal gravity waves associated with seismic activity, in: Seismo Electromagnetics: Lithosphere-Atmosphere-Ionosphere Coupling (Eds. M. HaYakawa and O. Molchanov) TERRAPUB, Tokyo, 249-253.

Molchanov, O.A.(2004): On the origin of low- and middle-latitude ionospheric turbulence, Phys. Chem. Earth., 29, 559-567.

Molchanov, O.A. and M. HayaKawa (1998): Subionospheric VLF signal perturbations possibly related to earthquakes, J. Geophys. Res., 103, 17.489-17.504.

Molchanov, O.A., A. Rohzhnoi, M. Solovieva, O. AKentieva, J.J. Berthelier, M. Parrot, F. Lefeuvre, P.F. Biagi, L. Castellana and M. Hayakawa (2006): Global diagnostic of the ionospheric perturbations related to the seismic activity using the VLF radio signals collected on the DEMETER satellite, Natural Hazards and Earth System Sciences, 6, 745-753.

Morgounov, V.A., T. ONDOH and S. NAGAI (1994): Anomalous variation of VLF signals associated with strong earthquakes $(\mathrm{M} \geq 7.0)$, in Electromagnetic Phenomena Related to Earthquake Prediction, edited by $\mathrm{M}$. HAYAKAWA and Y. FuJINAWA (Terrapub, Tokyo), 409-428.

Rotheram, S. (1981a): Ground wave propagation, Part 1: Theory for short distances, IEE Proc. F. Commun. Radar Signal Process, 128, (5), 275-284.
RothERAM, S. (1981b): Ground wave propagation, Part 2:Theory for medium and long distances and reference propagation curves, IEE Proc. F. Commun. Radar Signal Process, 128 (5), 285-295.

Rozhnol, A.A., M.S. Solovieva, O.A. Molchanov and M. HAYAKAWA (2004): Middle latitude LF (40 kHz) phase variations associated with earthquakes for quiet and disturbed geomagnetic conditions, Phys. Chem. Earth, 29, 6589-598.

Rozhnol, A.A., M.S. Solovieva., O.A. Molchanov, M. HaYAKaWA, S. MaEKaWA and P.F. Biagi (2005): Anomalies of LF signal during seismic activity in NovemberDecember 2004, NHESS, 5, 657-660.

Rozhno,, A.A., M.S. Solovieva., O.A. Molchanov, V. Chebrov, V. Voropaev, M. Hayakawa, S. Maekawa and P.F. BIAGI (2006): Preseismic anomaly of LF signal on the wave path Japan-Kamchatka during NovemberDecember 2004, Phys. Chem. Earth, 31, 422-427.

RuE, O. (1987): A new clear air interference prediction method for small time percentages, ICAP-87, Conf. Publ. No. 274, 343-346.

Strang, G. and T. Nguye (1996): Wavelets and Filter Banks (Wellesley Cambridge Press), pp. 490.

TElford, W.M., L.P. GELDART and R.E. SheRIFF (2002): Electric properties of rocks and minerals, in Applied Geophysics (Cambridge University Press, New York), 283-292.

Torrence, C. and G.P. Compo (1998): A practical guide to wavelet analysis, Bull. Amer. Meteorol. Soc., 79 (1), 61-78. 